\title{
Exploiting the Potential Energy Landscape to Sample Free Energy
}

\author{
Andrew J. Ballard, ${ }^{1, *}$ Stefano Martiniani, ${ }^{1, \dagger}$ Jacob D. \\ Stevenson, ${ }^{1, \ddagger}$ Sandeep Somani, ${ }^{1,2, \S}$ and David J. Wales ${ }^{1, \uparrow}$ \\ ${ }^{1}$ Department of Chemistry, Lensfield Road, Cambridge CB2 1EW, UK \\ ${ }^{2}$ Janssen Research and Development, LLC 1400 McKean Road, Spring House, PA 19477, USA \\ (Dated: February 16, 2015) \\ Abstract
}

*ab2111@cam.ac.uk

$\dagger$ †s958@cam.ac.uk

¥js850@cam.ac.uk

§ssomanilits.jnj.com

『dw34@cam.ac.uk 


\section{INTRODUCTION}

In this contribution we provide an overview of recent methods that all have their basis in enhancing the sampling of global thermodynamics using knowledge of the underlying potential energy landscape. Making these connections seems particularly timely, as a number of potentially important advances have been made over the last few years, and they share some common characteristics. In particular, the use of low-lying local minima obtained from methods based on global optimisation ( $\S$ III), and the superposition approach ( $\S$ II), provide some common themes. Of course, many other methods have been proposed for enhanced sampling [1-13], and these will not be reviewed here. The procedures we describe, with foundations in potential energy landscape theory [14-16] and geometry optimisation, are largely complementary to approaches based on more conventional molecular dynamics and Monte Carlo schemes [17]. Future work will require benchmarking comparisons of the most promising methods, to guide applications in different fields. For the present purposes our main aim is to show what has been achieved in recent work based on the potential energy landscape framework, and explain how these new tools are connected.

Most of the tests we have conducted employ atomic clusters of $N$ atoms bound by the LennardJones (LJ) potential [18]

$$
V=4 \epsilon \sum_{i<j}\left[\left(\frac{\sigma}{r_{i j}}\right)^{12}-\left(\frac{\sigma}{r_{i j}}\right)^{6}\right],
$$

where $\epsilon$ and $2^{1 / 6} \sigma$ are the pair equilibrium well depth and separation, respectively. These systems will be denoted $\mathrm{LJ}_{N}$, and there are a number of sizes where low temperature solid-solid phase transitions have been identified in previous work [7, 8, 14, 19-26]. In each case there are competing low energy morphologies separated by potential energy barriers that are large compared to $k_{B} T$ at the transition temperature. The relatively simple potential makes these systems ideal benchmarks for analysis of broken ergodicity issues, as well as for global optimisation [14, 19, 27] and rare event dynamics [28-31]. In principle, the same improvements would be obtained for treatments that explicitly consider electronic structure. However, the much greater computational expense of computing energies and gradients that would be necessary mean that these opportunities have yet to be exploited.

We begin by introducing the superposition approach $(\S$ II), a key component in the methods under review, which allows us to link thermodynamics to the basins of attraction of local minima on the underlying energy landscape. With this background in place, we review in $\S$ III the basin- 
hopping strategy for global optimisation. We then consider strategies for thermodynamic estimation, including replica exchange ( $(\mathrm{IV})$, Kirkwood sampling $(\S \mathrm{V})$, nested sampling $(\S \mathrm{VI})$, and basin-sampling ( $\S$ VII). In these sections we emphasize the utility of the superposition approach in enhancing these simulations, and provide evidence for gains in efficiency and convergence from simulation results for benchmark systems.

\section{THE SUPERPOSITION APPROACH}

A common theme in several of the following sections is the superposition approach to thermodynamics, which represents a key component of the computational potential energy landscape framework [14]. Here we write the total partition function or density of states as a sum over contributions from the basins of attraction $[14,32,33]$ of the local minima, which gives an explicitly ergodic representation of the thermodynamics [8, 14, 34-37]. As a first approximation it is often convenient to use harmonic vibrational densities of states for the local minima. The resulting superposition partition function is nevertheless anharmonic, owing to the distribution of local minima in energy. We can therefore separate contributions to thermodynamic properties in terms of the individual potential wells and the sum over minima. For example, we can identify well and landscape anharmonicity [38, 39]. For low temperature solid-solid equilibria the harmonic normal mode approximation can be quantitatively accurate, but methods to sample anharmonicity are generally required if an accurate picture of melting transitions is needed. Reweighting schemes and temperature-dependent frequencies have been considered for this purpose in previous work [35, 40-43]. The most recent basin-sampling scheme [44] will be outlined in $\S$ VII.

The underlying superposition representation for the canonical partition function is

$$
Z(T)=\sum_{\alpha=1}^{N^{\mathrm{st}}} \sum_{\zeta=1}^{N_{\alpha}^{\mathrm{PI}}} Z_{\zeta}(T)=\sum_{\alpha=1}^{N^{\mathrm{st}}} N_{\alpha}^{\mathrm{PI}} Z_{\alpha}(T)=P \sum_{\alpha=1}^{N^{\mathrm{st}}} Z_{\alpha}(T) / o_{\alpha},
$$

where $Z(T)$, is decomposed in terms of contributions from the catchment basin [14, 32] of each of the $N^{\text {st }}$ distinct local minimum structures, with $Z_{\alpha}(T)$ the partition function of structure $\alpha$ at temperature $T$, which is identical for each of the corresponding $N_{\alpha}^{\mathrm{PI}}$ permutation-inversion isomers. Here $P$ is the number of permutation-inversion operations of the Hamiltonian, $2 \prod_{\beta} N_{\beta}$ !, and $o_{\alpha}$ is the order of the rigid molecule point group [14, 45-47].

The great advantage of the superposition approach is that we can exploit efficient methods for locating low-lying local minima based on global optimisation, as described in $\S$ III. Basin- 
hopping approaches that incorporate a local minimisation [19, 48, 49] can employ arbitrary moves through configuration space, which can circumvent the barriers that cause ergodicity breaking in standard Monte Carlo and molecular dynamics procedures. Superposition methods employing both harmonic and anharmonic approximations of the vibrational density of states have found widespread applications in molecular science, and various examples are considered in the following review. First we outline the basin-hopping global optimisation approach, which has been used to obtain most of the samples of low-lying potential energy minima. We note that basin-hopping is a stochastic procedure, so it is not guaranteed to find all the relevant low-lying minima. However, the sampling of local minima, which does not require detailed balance, is much faster than for thermodynamic sampling approaches, and is straightforward for the systems considered here.

\section{BASIN-HOPPING GLOBAL OPTIMISATION}

The thermodynamic sampling schemes described in the following sections are of particular interest for systems involving broken ergodicity at low temperature, where the potential energy landscape supports alternative low-lying morphologies separated by relatively high barriers. Here, a 'high' barrier corresponds to a value that is large compared to $k_{B} T$ at the temperature, $T$, where the structures would have equal occupation probabilities, and $k_{B}$ is Boltzmann's constant. Basinhopping $(\mathrm{BH})$ global optimisation $[19,48,49]$ has been successfully employed to survey lowlying minima in a wide range of systems of this type, including atomic and molecular clusters, biomolecules, and soft and condensed matter [14, 49, 50]. These applications will not be reviewed here; since the $\mathrm{BH}$ procedure is now well established an overview should be sufficient in the present context.

The key point of the basin-hopping framework is to couple local energy minimisation to some sort of step-taking procedure in configuration space. Random perturbations of the atomic coordinates sometimes work quite well, but more efficient schemes can often be devised, which may exploit characteristics of the system under consideration. For example, internal coordinate moves, such as Kirkwood sampling, that respect connectivity are likely to work better for biomolecules with a well-defined covalently bonded framework. Numerous BH variants with alternative steptaking schemes have now been described, and parallel approaches using replicas at different effective temperatures have also been used [51]. In each case the potential energy for any configuration, $\mathrm{X}$, becomes the potential energy of the local minimum that the chosen minimisation procedure 
converges to from $\mathbf{X}$ :

$$
\widetilde{V}(\mathbf{X})=\min \{V(\mathbf{X})\}
$$

where $\mathrm{X}$ is a $3 N$-dimensional vector for a system of $N$ atoms. The resulting minimum replaces the previous structure in the chain if it satisfies a chosen acceptance condition, and again several alternatives have been considered. A simple Metropolis scheme often works well, where the new minimum with potential energy $E_{\text {new }}$ is accepted if it lies below the potential energy of the starting point, $E_{\text {old }}$. If $E_{\text {new }}>E_{\text {old }}$ it is accepted if $\exp \left[\left(E_{\text {old }}-E_{\text {new }}\right) / k T\right]$ is greater than a random number drawn from the interval [0,1]. Schemes based on thresholding [52], downhill-only moves [53] and non-Boltzmann weights [54] based on Tsallis statistics [55, 56] have also been described.

Much larger steps in configuration space can be taken than for conventional molecular dynamics or Monte Carlo methods, since the energy becomes the value after minimisation, and there is no requirement to satisfy detailed balance. Downhill barriers are therefore removed, and atoms can pass through each other. In fact, there is a more subtle effect, which facilitates sampling when broken ergodicity is prevalent. The occupation probabilities of competing regions of configuration space for the transformed landscape $\widetilde{V}(\mathbf{X})$ have been found to overlap significantly over a wider temperature range, where moves still have a good chance of being accepted $[57,58]$.

\section{REPLICA EXCHANGE APPROACHES}

The local minima provided by $\mathrm{BH}$ can be very helpful to understand systems exhibiting broken ergodicity. However, if thermodynamic properties are required, then configurations within the vicinity of each minimum become important, and increasingly so with higher temperature. At temperatures where well anharmonicity becomes noticeable, alternative techniques are required for sampling the thermodynamic state of interest. In contrast to $\mathrm{BH}$, thermodynamic sampling techniques generally require detailed balance to be satisfied in order to ensure the correct canonical distribution is preserved. While many long-standing strategies exist for sampling stationary distributions, the most notable being Monte Carlo and thermostatted molecular dynamics algorithms, the restriction of detailed balance often renders such techniques inefficient or unpractical. For instance, a Monte Carlo simulation with random perturbation moves often requires a small displacement step in order to achieve a useful acceptance probability. As a result, the system only explores its local configuration space on shorter timescales, with large-scale rearrangements, which dominate simulation convergence, occurring on much larger timescales. This quite generic 
problem necessitates advanced sampling strategies.

Replica exchange $[59,60]$ (REX) has emerged as a promising technique to sample such systems exhibiting complex energy landscapes. REX refers to a family of methods in which $M$ independent copies or "replicas" of a system are simulated in parallel, typically at different temperatures, with occasional moves that attempt to swap configurations between neighboring replicas. While large energy barriers may prevent the system from exploring its configuration space at low temperatures, enhanced thermal fluctuations provide more rapid barrier crossings at high temperatures. By means of the swap attempts, the low-temperature replicas are exposed to the wider reaches of configuration space explored by the the high-temperature replicas, providing a means to sample on both sides of the barrier without directly overcoming it. In this section we will focus primarily on parallel tempering (PT) in which the replicas are defined by a progression of temperatures, but methods with differing Hamiltonians exist as well [61].

The REX moves must satisfy detailed balance, which is enforced through an acceptance probability for the exchange attempt. For two replicas, $A$ and $B$, having the same potential energy function $V$ but at different temperatures $T_{A}$ and $T_{B}$, the acceptance probability takes the form

$$
P_{\text {acc }}(\mathbf{X}, \mathbf{Y})=\min \left\{1, e^{\Delta \beta \Delta V}\right\}
$$

Here $\Delta \beta=1 / k_{B} T_{B}-1 / k_{B} T_{A}$, and $\Delta V=V(\mathbf{Y})-V(\mathbf{X})$ is the difference in potential energy of the swap configurations $\mathrm{X}$ and $\mathrm{Y}$ in question. Eq. 4 guarantees that each replica samples its correct equilibrium state, despite the fact that configurations are being swapped between different thermodynamic ensembles.

Just as detailed balance restricts the perturbation stepsize in a MC simulation, in REX it limits the temperate spacings between neighboring replicas. To ensure a decent average acceptance rate $\left\langle P_{\text {acc }}\right\rangle$ the temperatures $T_{A}$ and $T_{B}$ must be spaced closely, yet still far enough apart not to waste computational resources on an unnecessary number of replicas. This conflict has given rise to numerous strategies to optimize various PT parameters, in particular the temperature spacings and total number of replicas.

Understanding a system in terms of the underlying energy landscape can be particularly useful in this respect, and recent developments have been made that enhance PT simulations by utilizing information contained in the energy landscape. These developments, described below, exploit the harmonic superposition approximation (HSA), a harmonic expansion of the landscape about the known configurational minima. Through this approximation, an approximate energy landscape 
can be constructed solely from a set of minima, and since the form is particularly convenient, namely a series of multi-dimensional harmonic wells, thermodynamic predictions can be easily made. In this way, thermodynamics from the HSA can be used to approximate the behaviour of the true underlying system.

The HSA can be exploited to determine optimal PT temperature spacings, a crucial component in the performance of PT simulations. In general a uniform acceptance profile is desired across all neighboring replicas [62-67], which guarantees that replica round-trip times are not plagued by bottlenecks as trajectories transition between temperatures. Ballard and Wales [68] have recently demonstrated how knowledge of configurational minima can be utilized to optimize PT temperatures in this respect. By approximating the canonical distribution of each replica via the HSA, they obtained analytic expressions for the average acceptance rate $\left\langle P_{\text {acc }}\right\rangle$ in terms of features of the configurational minima. From these expressions, a set of optimal temperatures can be uniquely determined by matching to a target acceptance rate, yielding a progression of replicas that has a uniform acceptance within the approximation of the HSA. Simulations of systems undergoing phase transformation have revealed that this strategy can yield uniform acceptance rates and efficiency enhancements over a standard progression. Fig. 1 displays the uniform acceptance profile achieved by the method on PT simulations of $\mathrm{LJ}_{31}$.

In addition to optimizing temperature spacings, the HSA can also be used as a replica in itself. Mandelshtam and coworkers [7, 8] have devised a REX strategy whereby an auxiliary "reservoir" replica is coupled to an $M$-replica PT simulation. The reservoir replica samples the HSA at a user-specified temperature, with reservoir-temperature swap moves occurring with a particular temperature replica. Because HSA samples can be drawn analytically, reservoir sampling is essentially barrierless which enables rapid exploration of the system's relevant configuration space (defined by the known minima). In this way the reservoir reduces the need for high temperature replicas to overcome barriers. Similar strategies for MC sampling employ analogous ideas by generating trial moves near known configurational minima $[1,5]$.

\section{KIRKWOOD SAMPLING}

Kirkwood sampling [69] is a method for random (or non-Markovian) sampling of the conformational space of molecules. The principal challenge in random conformational sampling is to avoid steric clashes among the atoms. Kirkwood sampling addresses this problem by incorporating 


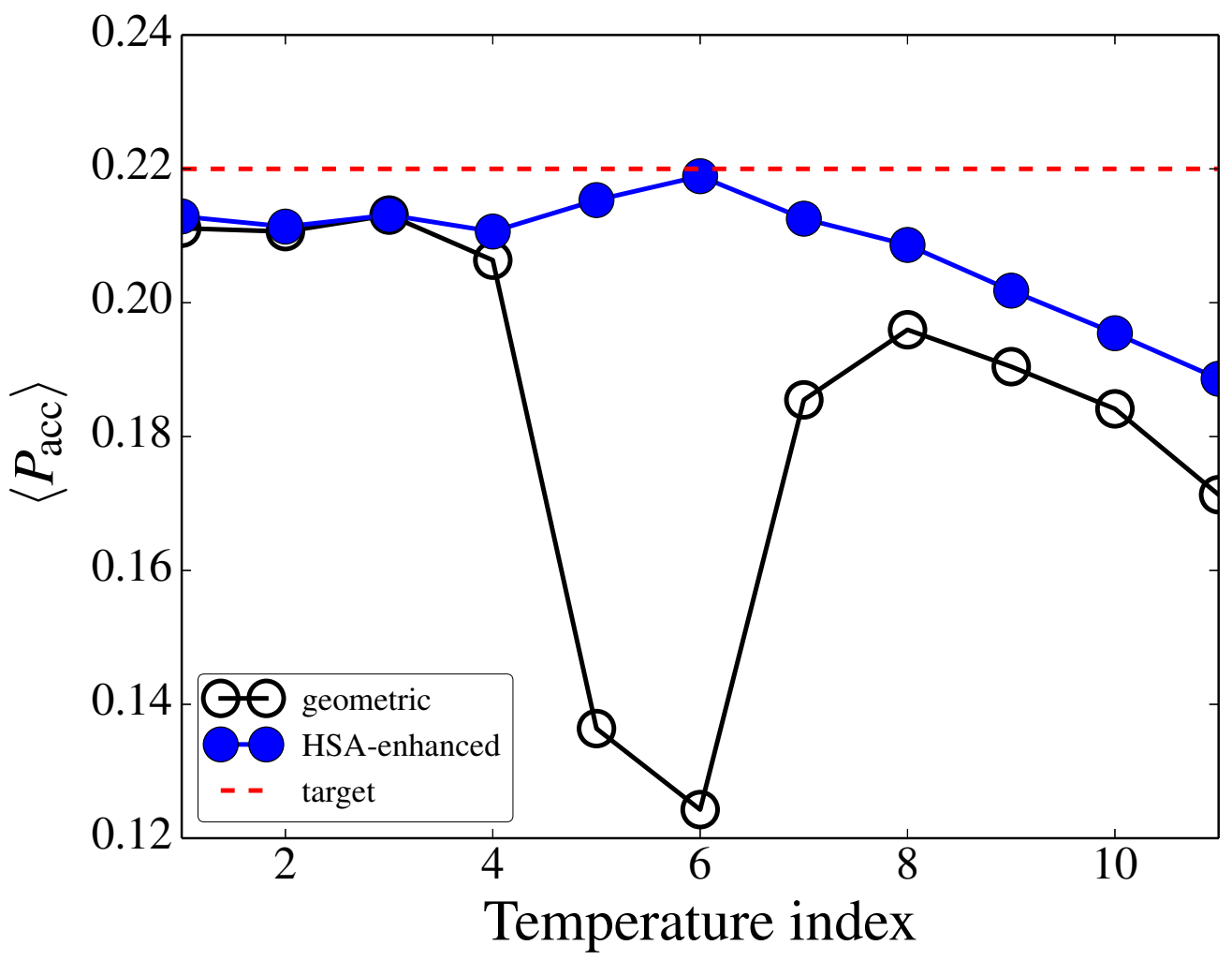

FIG. 1: Acceptance profile for REX simulation of $\mathrm{LJ}_{31}$ : The average REX acceptance probability for pair $\left(T_{i}, T_{i+1}\right)$ is plotted vs temperature index $i$, for temperature spacings chosen using standard geometric progression (empty black circles), and chosen by HSA-optimization (filled blue circles). The dip in the geometric case coincides with a heat capacity peak. At higher temperatures both profiles deviate from uniform behavior, as the HSA becomes less accurate. Results replotted from Ref. [68]

correlations among internal coordinates, as captured by the joint probability distribution function (pdf) between various sets of internal coordinates. Kirkwood sampling is based on the generalization of the Kirkwood superposition approximation (KSA), originally developed in the radial distribution function theory of liquids $[70,71]$. Analogous approximations were later developed in the mutual information theory of correlations and provided expressions for a multidimensional pdf in terms of its marginal pdfs corresponding to neglect of certain correlations. The current application to conformational sampling was motivated by conformational entropy calculation of small molecules using the mutual information expansion of entropy [72, 73]. The simplest approximation in this family, namely the Kirkwood superposition approximation, $p_{3}^{K S A}$, expresses a three-dimensional pdf, $p_{3}$, in terms of its one- and two-dimensional marginal pdfs

$$
p_{3}\left(x_{1}, x_{2}, x_{3}\right) \approx p_{3}^{K S A}\left(x_{1}, x_{2}, x_{3}\right)=\frac{p_{2}\left(x_{1}, x_{2}\right) p_{2}\left(x_{1}, x_{3}\right) p_{2}\left(x_{2}, x_{3}\right)}{p_{1}\left(x_{1}\right) p_{1}\left(x_{2}\right) p_{1}\left(x_{3}\right)}
$$


where $p_{1}($.$) and p_{2}(.,$.$) are the marginal pdfs, and the subscript indicates the dimensionality of the$ pdf. Eq. 5 can be obtained from the mutual information expansion of Shannon entropy of $p_{3}$ by dropping the three-fold mutual information $[69,74]$. The Kirkwood superposition approximation can be generalized to express an $N$-dimensional pdf in terms of its marginals of highest order $l$ [74]. For instance, the doublet level $(l=2)$ level superposition approximation is given by the ratio of the product of 2-D and 1-D marginal pdfs

$$
p_{N} \approx p_{N}^{(2)}\left(x_{1}, \ldots, x_{N}\right)=\frac{\prod_{i<j} p_{2}\left(x_{i}, x_{j}\right)}{\prod_{i} p_{1}\left(x_{i}\right)}
$$

where the superscript "(2)" denotes doublet level. Eq. 6 accounts for pairwise correlations among all variables but ignores the higher order correlations. Approximations that account for selected correlations of different orders can also be derived [75]. Eq. 6 is fully coupled and therefore exact sampling would be computationally prohibitive for high-dimensional systems. The Kirkwood approach addresses this issue by sequential sampling of the variables such that each one is selected from a one-dimensional conditional distribution conditioned on the previously sampled variables. The conditional distributions are obtained using the appropriate superposition approximation. For example, given a sampling order, the conditional pdf for the $k$-th variable given values of previous $k-1$ variables, using the doublet level approximation (Eq. 6), is

$$
\begin{aligned}
p_{1}^{(2)}\left(x_{k} \mid x_{1}, . ., x_{k-1}\right) & =\frac{p_{k}^{(2)}\left(x_{1}, . ., x_{k-1}, x_{k}\right)}{p_{k-1}^{(2)}\left(x_{1}, . ., x_{k-1}\right)} \\
& =\frac{1}{n_{k}} \frac{\prod_{1 \leq j \leq k-1} p_{2}\left(x_{j}, x_{k}\right)}{p_{1}\left(x_{k}\right)^{k-2}}
\end{aligned}
$$

where the normalization $n_{k}$ can be computed numerically. The doublet level Kirkwood scheme effectively samples from the $N$-dimensional pdf

$$
\widetilde{p}_{N}^{(2)}(\vec{x})=p_{2}\left(x_{1}, x_{2}\right) \prod_{3 \leq k \leq N} p_{1}^{(2)}\left(x_{k} \mid x_{1}, x_{2}, . ., x_{k-1}\right)
$$

and not from Eq. 6. Thus, at the doublet level, the sequence of approximations for the true distribution, $p^{N}$, is

$$
p_{N} \rightarrow p_{N}^{(2)} \rightarrow \widetilde{p}_{N}^{(2)}
$$

Note that both the doublet level Kirkwood approximation, $p_{N}^{(2)}$, and the doublet level sampling distribution, $\widetilde{p}_{N}^{(2)}$, involve a product of all singlet and doublet pdfs. Due to this product form, a sampled conformation is guaranteed to fall in the non-zero probability cells of all input pdfs. 
This construction ensures that all correlations are satisfied simultaneously. The computational complexity of a Kirkwood sampling algorithm is proportional to the number of pdfs used. Since the number of doublet level marginals are $N(N+1) / 2$, the doublet level sampling algorithm has the complexity of $O\left(N^{2}\right)$.

Doublet and triplet level Kirkwood sampling has been applied to small drug-like molecules [69] and small peptides [76] with up to 52 atoms. In these studies, bond-angle-torsion [77] internal coordinate system was used and the input pdfs were populated using high temperature MD simulation data and were effectively marginal pdfs of the Boltzmann distribution at the simulation temperature. Figure 2 shows that the majority of the conformations generated by doublet level sampling for alanine tetrapeptide $(N=150)$ had energies similar to those of conformations sampled by the original MD simulation. In other words, the doublet level Kirkwood sampling distribution is a good approximation of the original Boltzmann distribution. These studies suggest that accounting for just the low order correlations is sufficient for avoiding steric clashes at the local level, for example, between adjacent residues of a peptide.

We note that due to high dimensionality and neglect of the higher order correlations the conformational space accessible to Kirkwood sampling will be much larger than that sampled in the original MD simulation. In Figure 2, this observation is reflected in the shift of the Kirkwood energy distribution to higher energies since more conformations are available at higher energies. Unlike MD simulations, Kirkwood sampling generates uncorrelated samples. Furthermore, it is a geometrical sampling method, independent of the potential energy function, and therefore provides barrierless global sampling for any potential energy surface.

In the context of biomolecular simulations, performance of other methods described in this contribution can be enhanced by employing Kirkwood sampling for step taking. For enhanced sampling of local minima Kirkwood samples could be used for seeding independent basin-hopping simulations. Kirkwood samples can also be used for generating the initial replicas for nested sampling simulations. Since the normalized probability of generating a Kirkwood sample is available, a key advantage over other, knowledge-based conformational samplers [78], Kirkwood sampling can also be combined with thermodynamic sampling algorithms that require detailed balance to be satisfied. We next discuss two approaches [79] for obtaining Boltzmann distributed conformations from Kirkwood sampling.

The first approach utilizes the biased Monte Carlo [17] framework with the Kirkwood sampling distribution as the biasing distribution. Here Kirkwood samples are used as trial moves and 
the Metropolis acceptance function is modified to reweight according to the Boltzmann distribution. For example, if doublet level Kirkwood sampling is used (Eq. 8), the Metropolis acceptance function is given by

$$
P_{a c c}\left(\mathbf{X}_{n}, \mathbf{X}_{o}\right)=\min \left(1, \frac{e^{-\beta U\left(\mathbf{X}_{n} ; \beta\right)} / \widetilde{p}_{N}^{(2)}\left(\mathbf{X}_{n}\right)}{e^{-\beta U\left(\mathbf{X}_{o} ; \beta\right)} / \widetilde{p}_{N}^{(2)}\left(\mathbf{X}_{o}\right)}\right),
$$

where $\mathbf{X}_{n}$ is the new trial conformation and $\mathbf{X}_{o}$ is the old conformation. The energy $U\left(\mathbf{X}_{n} ; \beta\right)$ includes a contribution due to the Jacobian, $J(\mathbf{X})$, of transformation from internal to Cartesian coordinate system

$$
U(\mathbf{X} ; \beta) \equiv V(\mathbf{X})-\frac{1}{\beta} \ln J(\mathbf{X})
$$

where $V$ is the potential energy, typically specified by a molecular mechanics forcefield. In contrast to standard perturbation move MC algorithms, which are sequential, the moves in biased MC are independent of the current conformation. Consequently no equilibration is required and the algorithm can be trivially parallelized in a distributed computing environment. Furthermore, since Kirkwood sampling is a geometrical sampling approach, the same set of Kirkwood samples may be reweighted to generate Boltzmann distributions for different potential energy functions and temperatures.

We have applied [79] doublet Kirkwood biased MC simulation to a model system with nine atoms and a bonded chain topology. The input pdfs were generated using data from a $500 \mathrm{~K}$ MD simulation and biased MC simulations were performed at successively lower temperatures of $500 \mathrm{~K}, 400 \mathrm{~K}, 300 \mathrm{~K}$ and $200 \mathrm{~K}$. Biased MC simulations were able to generate Boltzmann distributions at the MD temperature as well as at lower temperatures, though the acceptance ratios fell with reduced temperatures, consistent with the lower overlap. One can imagine an iterative scheme where the initial set of pdfs is constructed in a manner that provides coverage of a wide conformational space. For instance, the pdfs could be populated using high temperature MD, or using a database of conformations from structural databases $[80,81]$ or a pdf library for molecular fragments. Given this initial set of pdfs and a potential energy function, one could potentially perform successive stages of biased MC simulations and repopulation of the pdfs to reach arbitrarily low temperatures. Note that, for a given potential energy function and temperature, the acceptance ratio of the Kirkwood biased MC simulation is completely determined by the input pdfs and provides a direct measure of the overlap between the Kirkwood sampling distribution and the target Boltzmann distribution. A given acceptance ratio would impose a lower limit on the temperatures for which biased MC simulations can be run. 
Due to the product form of the Kirkwood sampling distribution, if there are zero probability cells in the input pdfs then certain regions of the configurational space will not be accessible. As a result Kirkwood sampling will not be ergodic, although the eliminated regions are likely to correspond to conformations with atom clashes. For thermodynamic sampling, non-ergodicity can be compensated by combining Kirkwood sampling with Markovian samplers, such as MD, or perturbation move Monte Carlo which explore the conformational space in the vicinity of the current conformation. This exploration is accomplished using the Kirkwood sampler as a reservoir in a reservoir replica exchange simulation. The acceptance function for exchanging between Kirkwood reservoir and a temperature replica is given by

$$
P_{a c c}\left(\mathbf{X}_{\beta}, \mathbf{X}_{r}\right)=\min \left(1, \frac{\widetilde{p}_{N}^{(2)}\left(\mathbf{X}_{\beta}\right)}{\widetilde{p}_{N}^{(2)}\left(\mathbf{X}_{r}\right)} e^{\beta\left(U\left(\mathbf{X}_{\beta} ; \beta\right)-U\left(\mathbf{X}_{r} ; \beta\right)\right)}\right)
$$

where $\mathrm{X}_{\beta}$ is the conformation from a temperature replica and $\mathrm{X}_{r}$ is reservoir conformation, here drawn from a Kirkwood sampling distribution. The doublet Kirkwood distribution is used in Eq. 12 for illustration. Note that the acceptance function involves the reservoir probability of the conformation from the temperature replica as well as the potential energy of the reservoir conformation. Tests on the nine-atom model system show (Figure 4) improved convergence when a Kirkwood reservoir is employed.

As in the case of biased MC, the acceptance ratio for exchanges with the reservoir is determined by the overlap of the Kirkwood distribution and the Boltzmann distribution for the coupled temperature replica. Note that in the absence of a reservoir the highest temperature in a replica exchange simulation needs to be high enough to overcome the energy barriers and avoid trapping. By coupling to a Kirkwood reservoir the highest temperature would be dictated by the desired exchange acceptance ratio. Depending on the input pdfs used, Kirkwood dictated highest replica temperature may be lower than the highest temperature dictated by the barriers on the energy landscape. Indeed, if the reservoir has good overlap with the Boltzmann distribution corresponding to the temperature of interest, then just a single replica would suffice. In this case, the temperature replica essentially performs local sampling, while the reservoir facilitates global sampling of the conformational space. One can also imagine a replica exchange simulation where the highest temperature replica is coupled to a Kirkwood reservoir to facilitate global sampling and the lowest temperature is coupled to a HSA reservoir constructed from the low energy minima to enable rapid equilibration over the low energy regions of the energy landscape. 

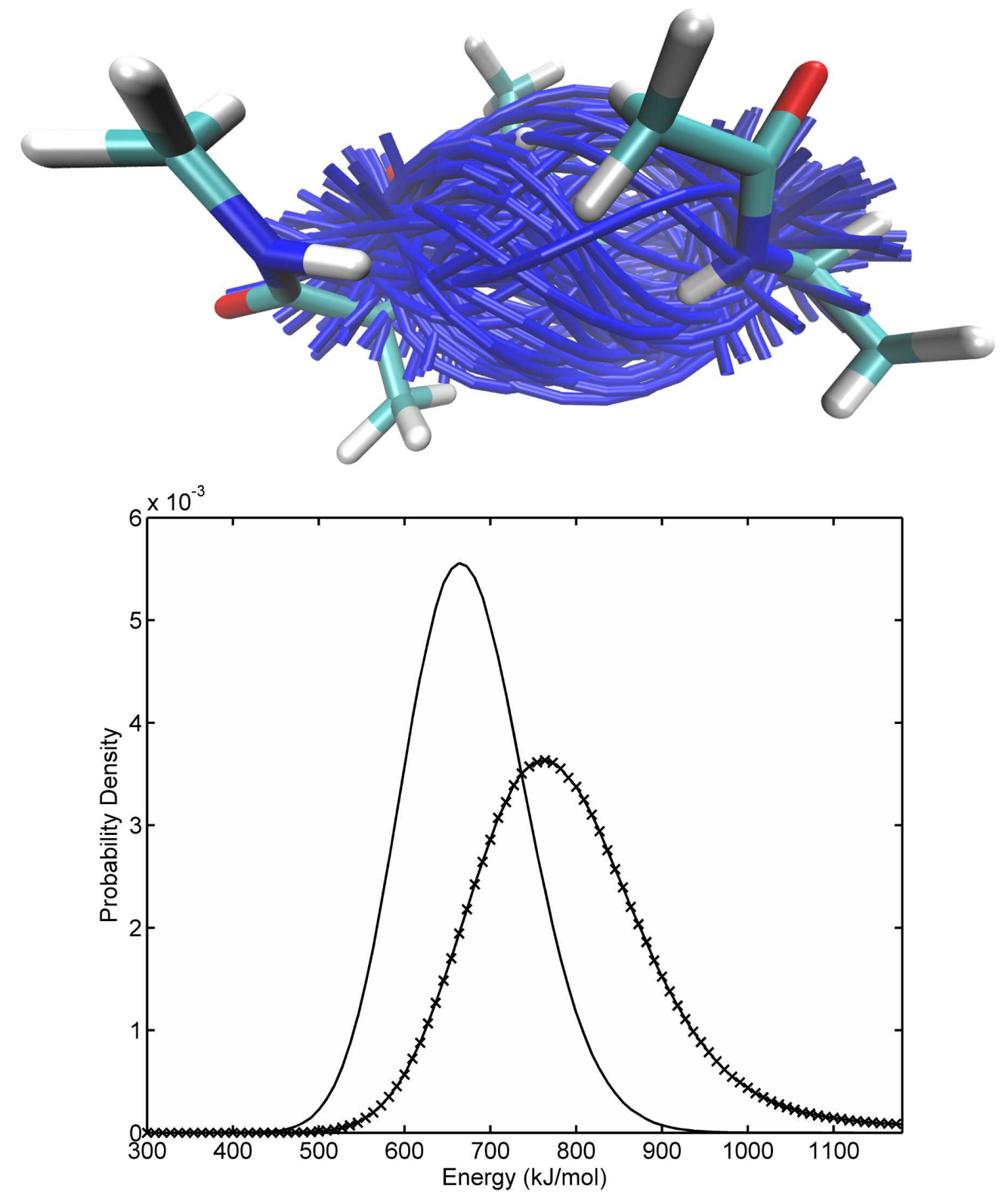

FIG. 2: Doublet level Kirkwood sampling results for 52-atom tetra alanine peptide. The input singlet and doublet pdfs were populated using 5 million conformations from a 500 ns vacuum MD simulation performed at $1000 \mathrm{~K}$. (Top) shows the backbone (blue tube) of 100 Kirkwood sampled conformations aligned on the backbone atoms; one conformation is also shown in licorice. Potential energy for a million Kirkwood samples was computed using the MD energy function. (Bottom) shows the energy distribution of the Kirkwood samples (crosses) overlaid on the Boltzmann energy distribution obtained from the MD simulation (unmarked). The two distributions have substantial overlap indicating overlap of the Kirkwood sampled and MD sampled conformational space. 


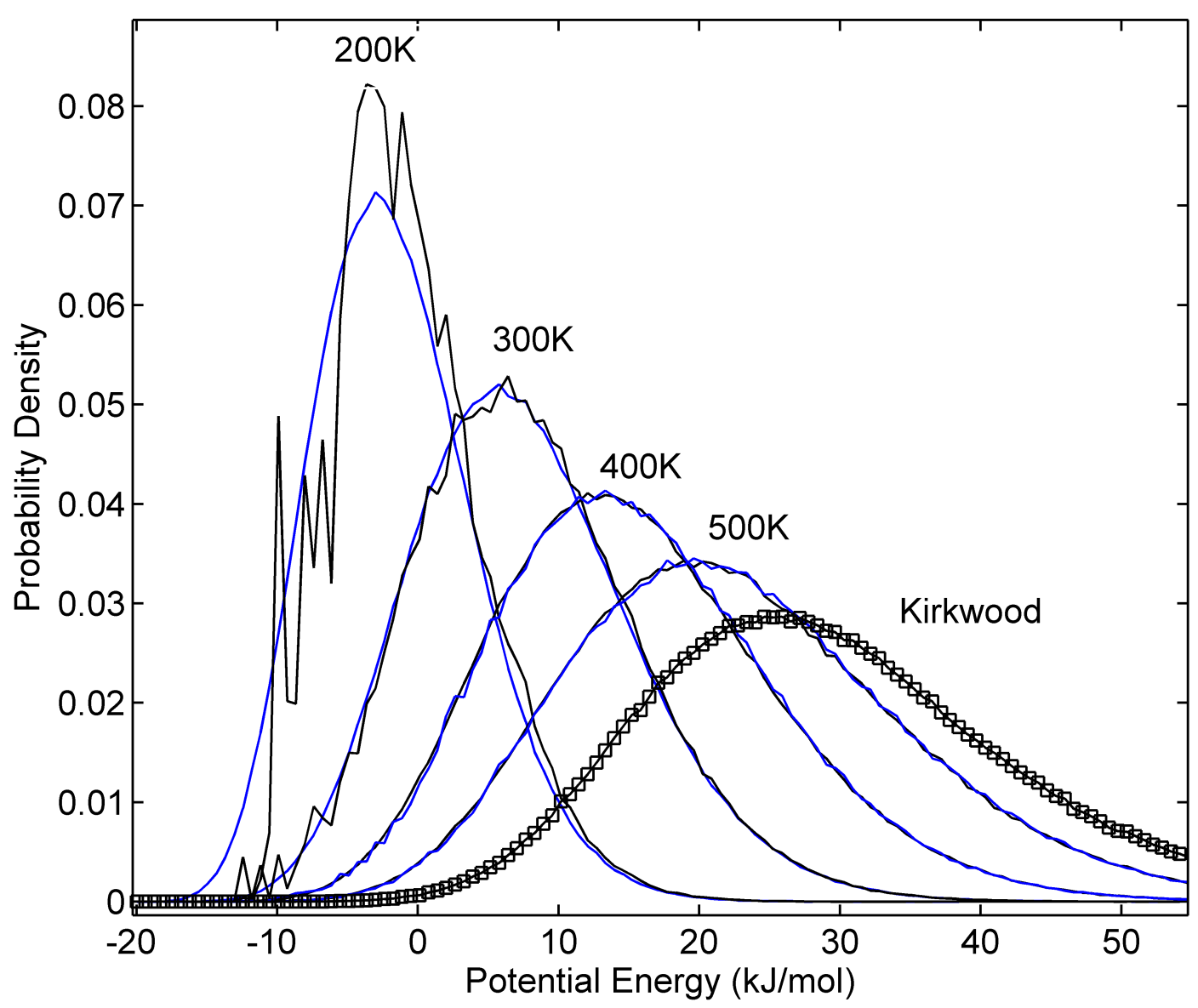

FIG. 3: Biased MC simulations for a 9-atom chain molecule using doublet level Kirkwood sampling distribution as the biasing distribution. Distribution of energy from one million step biased MC simulations (black unmarked line) performed at $T=500,400,300$ and $200 \mathrm{~K}$, is overlaid on the corresponding reference Boltzmann distributions (in blue unmarked lines). The distribution of energy of the Kirkwood samples is marked by boxes. The acceptance ratio for the different simulations were $0.29,0.25,0.07$ and 0.009 for $T=500,400,300$ and $200 \mathrm{~K}$, respectively. These results show that this approach can generate Boltzmann distributions even at temperatures lower than that of the original MD simulation used to populate the input pdfs

\section{SUPERPOSITION ENHANCED NESTED SAMPLING}

Nested sampling (NS) is an importance sampling method that was recently introduced in the Bayesian statistics community [82] and has since been applied in a variety of fields, including 

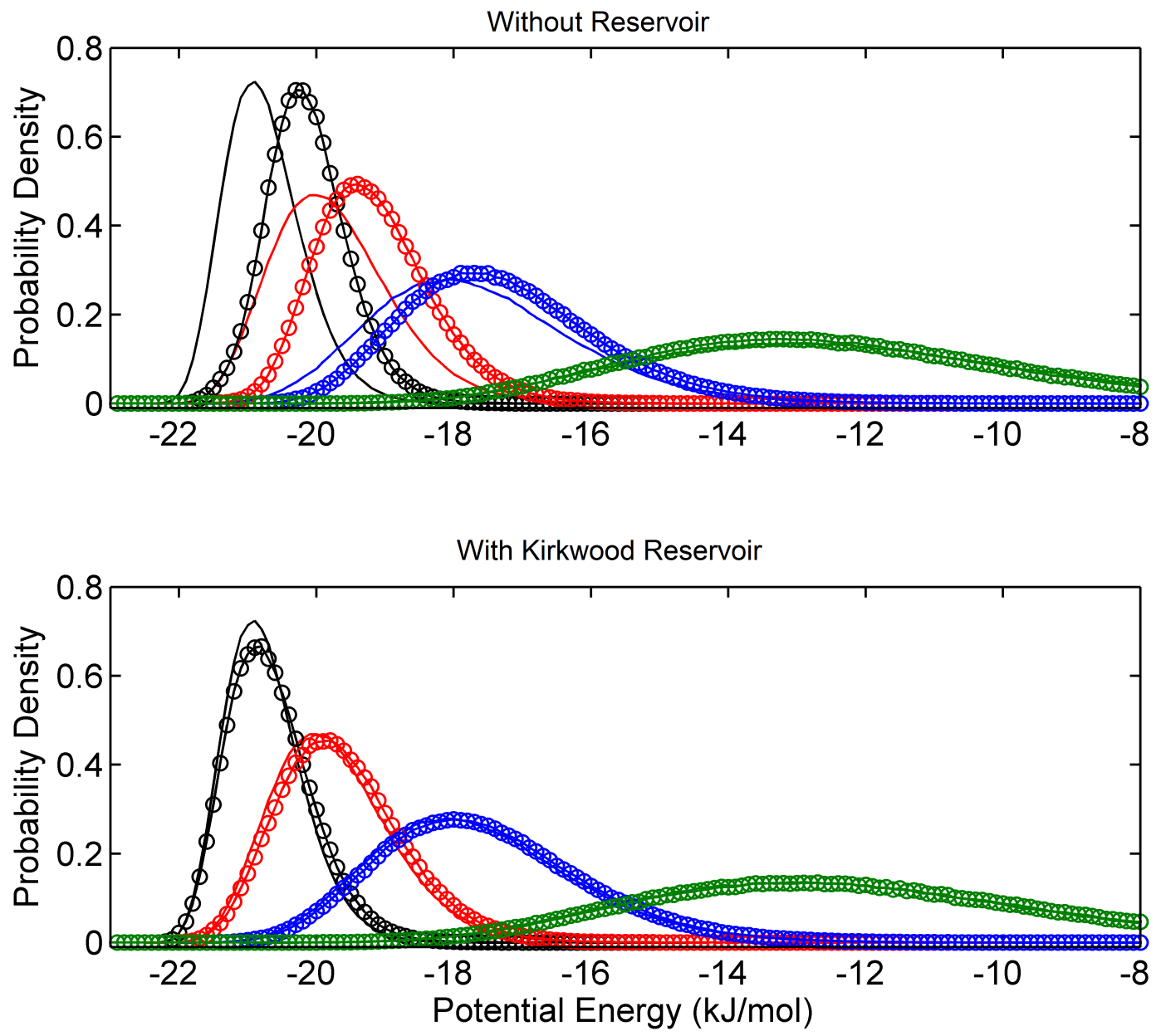

FIG. 4: (Color) Potential energy distributions (lines marked by circles) from the $5 \times 10^{6}$ steps temperature replica exchange simulations (a) without and (b) with a doublet level Kirkwood reservoir. The replica temperatures are $20 \mathrm{~K}$ (black), $30 \mathrm{~K}$ (red), $50 \mathrm{~K}$ (blue) and $100 \mathrm{~K}$ (green). The distributions from the Kirkwood reservoir simulation are much closer to the reference distributions (unmarked lines), indicating improved convergence upon coupling to a reservoir.

astrophysics [83, 84], systems biology [85], and statistical physics [86-88]. NS was originally described in the language of Bayesian statistics, but can equally be applied to thermodynamic sampling. The Bayesian prior is the uniform distribution over all of phase space (every point in phase space equally likely). The integral over phase space is performed, weighted by the likelihood, which is simply the Boltzmann weight $\exp (-V(\mathbf{X}) / T)$. In the execution of NS the value of the likelihood is unimportant; only the ordering matters, which means that the energy can be used as a proxy for the Boltzmann weight, making NS independent of temperature. The primary output 


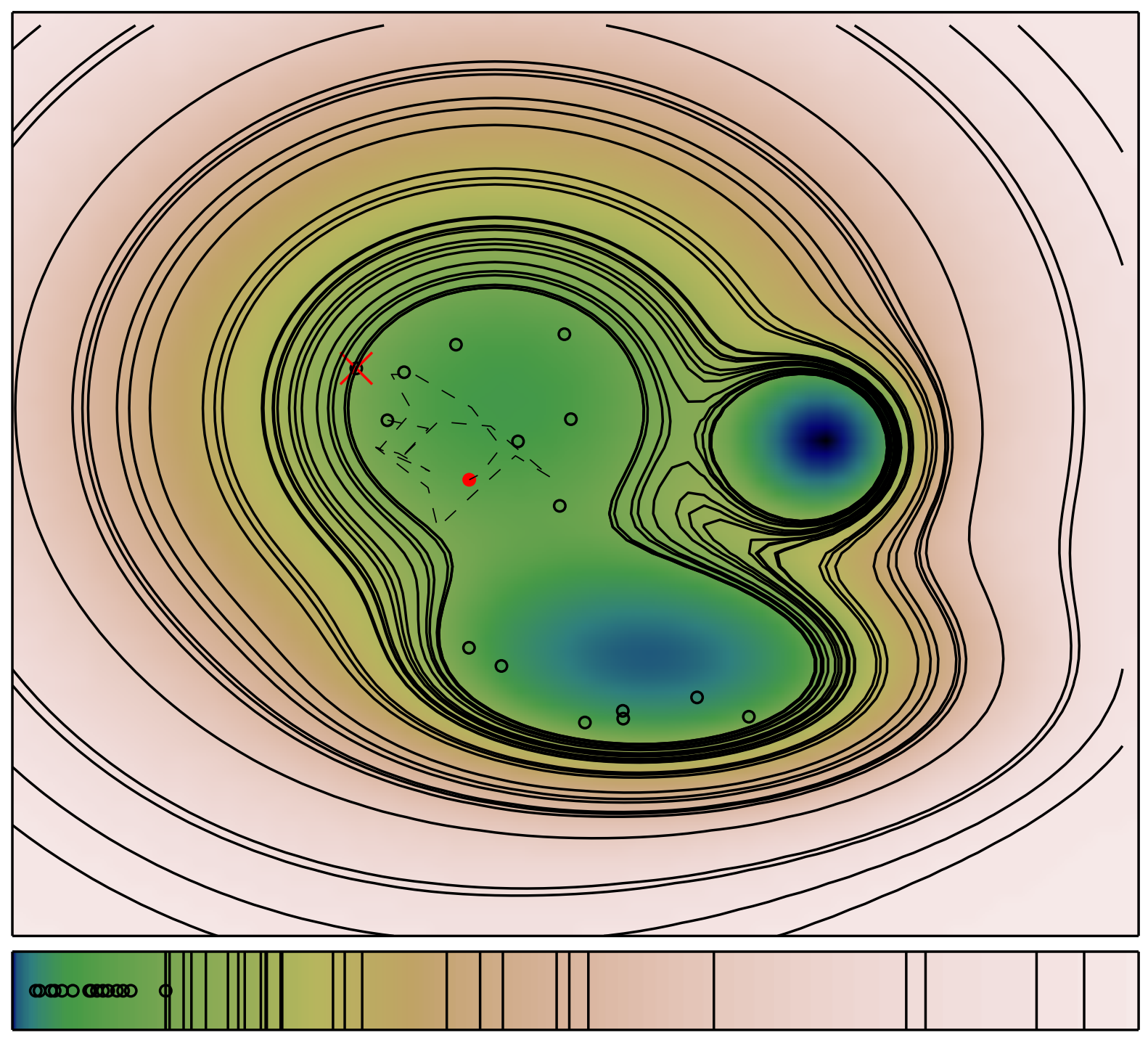

FIG. 5: The nested sampling procedure is illustrated for a two-dimensional energy landscape where darker colors indicate lower energies. The image is drawn after 26 iterations of the nested sampling procedure. The circles represent the replica positions $(\mathrm{K}=15)$, which are distributed uniformly in the two-dimensional space. The lines are constant energy contours at $E_{i}^{\max }$ for each iteration $i$. The cross gives the location of the highest energy replica, which defines $E^{\max }$ for the next iteration. The dotted line is the path of the MC walk of the newly generated replica, which is shown as a solid circle. The lower panel shows the one-dimensional representation of phase space, which is used to derive the compression factor of equations 13 and 15. In this configuration there are no replicas in the basin with the global minimum, which will cause the density of states to be overestimated. This major drawback of the NS algorithm can be overcome by superposition enhanced nested sampling if the basin-hopping procedure correctly identifies the relevant low-lying minima. 
of NS is the density of states, from which it is possible to compute thermodynamic quantities at any temperature.

Nested sampling proceeds by iteratively constructing a list of energy levels $\left\{E_{1}^{\max }, \ldots, E_{N}^{\max }\right\}$ which are sorted so that $E_{i}^{\max }>E_{i+1}^{\max }$. These energy levels have the special property that the volume of phase space with energy below $E_{i}^{\max }$ satisfies, on average

$$
\Omega\left(E<E_{i}^{\max }\right)=\alpha \Omega\left(E<E_{i-1}^{\max }\right)
$$

where $0<\alpha<1$ is determined by a parameter of the algorithm (equation 15). From this result, the density of states, $g_{i}$, the normalized phase space volume with energy between $E_{i}^{\max }$ and $E_{i-1}^{\max }$ is constructed as

$$
g_{i}=\frac{\Omega\left(E<E_{i-1}^{\max }\right)-\Omega\left(E<E_{i}^{\max }\right)}{\Omega_{0}}=\alpha^{i-1}-\alpha^{i},
$$

where $\Omega_{0}$ is the total phase space volume.

The nested sampling algorithm begins by generating $K$ configurations of the system (replicas) distributed randomly and uniformly within phase space $\Omega_{0}$. For systems with infinite phase space volume the system can be placed in a box and $\Omega_{0}$ is then the box volume. Alternatively, one can limit phase space by placing an upper bound on the energy. Either way, the actual value of $\Omega_{0}$ is only a normalization constant and does not come into the definition of the density of states. The energies of the replicas are computed and stored in a list $\left\{E_{1}, \ldots, E_{K}\right\}$. The key insight of nested sampling, is that the energy of the replica with the maximum energy $\left(E_{1}^{\max }\right)$ satisfies equation 13 as $\Omega\left(E<E_{1}^{\max }\right) \approx \alpha \Omega_{0}$ with the compression factor

$$
\alpha=\frac{K}{K+1} .
$$

This can be understood by laying out every point in phase space on a one dimensional line and sorting them by energy. The "volume" of the line is just the length, and goes from 0 (leftmost) to $\Omega_{0}$ (rightmost). The $K$ replicas are distributed randomly and uniformly on that line. The replica with the largest energy will be the rightmost replica. If $K$ points are placed on a unit line, the position of the point with the largest value will be distributed according to

$$
p_{K}(x) \sim x^{K-1}
$$

with expectation value $K /(K+1)$. Thus the mean position of the right-most replica is $\Omega_{0} K /(K+$ $1)$, which also corresponds to $\Omega\left(E<E_{1}^{\max }\right)$. 
We now remove the replica with the maximum energy and replace it with a new replica sampled uniformly from with the volume $\Omega\left(E<E_{1}^{\max }\right)$. This is typically done by initializing the new replica at the position of one of the others and walking it via a Monte Carlo Markov chain for sufficient steps that it loses its memory of the starting location. We now again have $K$ replicas sampled uniformly from the volume $\Omega\left(E<E_{1}^{\max }\right)$. Thus, using the same arguments as before, the energy of the maximum energy replica is saved as $E_{2}^{\max }$, and satisfies equation 13 . This procedure is iterated until some stopping condition is met.

A major benefit of nested sampling is that, although phase space is divided into energy bins, as in equation 14, the energy bins are determined adaptively via the constant compression factor $\alpha=K /(K+1)$. This stepping procedure automatically creates higher resolution for low energy regions of phase space and in regions where phase space volume is changing most rapidly, which usually applies near phase transitions. This feature of adaptively determined bin sizes is in contrast with the energy bins in the Wang-Landau method [3], and the temperature spacing in parallel tempering, which must be predetermined, although a method for choosing the temperature spacing was presented in IV. In fact, Brewer et. al [88] introduced diffusive nested sampling, which uses the energy bins and weights $g_{i}$ produced by NS as input to the Wang-Landau method to refine the estimate for the density of states.

The vast majority of the computation time in NS is spent generating new configurations sampled uniformly from the space $\Omega\left(E<E_{i}^{\max }\right)$. As discussed above, the standard way of achieving this goal is using a Monte Carlo walk long enough so that the replica loses memory of where it started. Uniform sampling is maintained by accepting every step subject only to the criterion that the new energy is less than $E_{i}^{\max }$. This technique can lead to serious sampling problems for multi-modal energy landscapes. If two minima have energy less than $E_{i}^{\max }$, but the minimum energy path between them lies above $E_{i}^{\max }$, then a MC walk started in one basin will never reach the other. This problem is partially alleviated by the fact that there are many independent replicas. As long as there are sufficient replicas in each basin then the statistics should not be affected too much. However if, through statistical fluctuations, one basin is devoid of replicas, it will never become repopulated. This issue is most likely to cause serious problems in systems where the basin with the global minimum is narrow and separated by large energy barriers from the rest of phase space. Examples of such difficult systems are $\mathrm{LJ}_{31}, \mathrm{LJ}_{38}$, and $\mathrm{LJ}_{75}$ [87].

New methods for uniform sampling with a likelihood (or energy) constraint are actively being developed [88]. Here we describe a recent method that uses ideas from potential energy landscape 
theory to speed up sampling and overcome the problem of being locked out of basins, namely superposition enhanced nested sampling (SENS) [89].

SENS combines the strength of global optimisation algorithms with that of NS to achieve an augmented exploration of the low energy regions of the energy landscape, while maintaining all the desirable properties of NS. Global optimisation algorithms, such as basin-hopping ( $\S$ III), are much more efficient at finding the low energy configurations by virtue of a greater freedom to use energy minimisation algorithms and Monte Carlo moves that do not respect detailed balance. The collection of minimum energy configurations thus obtained can be used to augment the exploration of the energy landscape performed by NS. SENS contributes knowledge of this collection of low energy configurations via Hamiltonian replica exchange (HREM) [90, 91]. Similar in spirit to PT, HREM moves are proposed between configurations sampled using different Hamiltonians (energy functions), rather than sampling configurations with the same Hamiltonian, but weighted at different temperatures, as in PT.

In this particular instance, similar in spirit to reference [91], a minimum is selected from the precomputed collection of minima weighted by their HSA configurational volume and a swap is proposed between a configuration $\mathbf{X}_{h a r}$, sampled uniformly within its harmonic basin of attraction, and a configuration $\mathbf{X}_{\text {sys }}$, sampled by a $\mathrm{MC}$ walk using the system Hamiltonian under a certain energy constraint $E_{\max }$. If

$$
H_{\text {har }}\left(\mathbf{X}_{\text {sys }}\right) \leq E_{\max } \text { and } H_{\text {sys }}\left(\mathbf{X}_{\text {har }}\right) \leq E_{\text {max }}
$$

then the true and the HSA distribution of states overlap and the swap is accepted. HREM moves are typically followed by conventional MC walks to further explore neighbouring regions of configurational space. The acceptance of such HREM moves depends heavily on how well the HSA approximates the underlying potential energy surface and so does the efficiency of SENS over that of NS. While the HSA captures landscape anharmonicity, arising from the distribution of local minima, it does not include well anharmonicity, thus becoming an increasingly good approximation at lower energies. For this reason we expect that only the low energy minima will contribute significantly to augmenting the sampling.

An example of a very successful application of SENS is found for the thermodynamic analysis of $\mathrm{LJ}_{31}$, where the method has been shown to be at least one order of magnitude more efficient that PT and NS [89]. In Fig. 6 we show results for the heat capacity curve of $\mathrm{LJ}_{31}$; for $K=20000$ replicas SENS is well converged and agrees with the HSA at low temperatures, while for NS the 


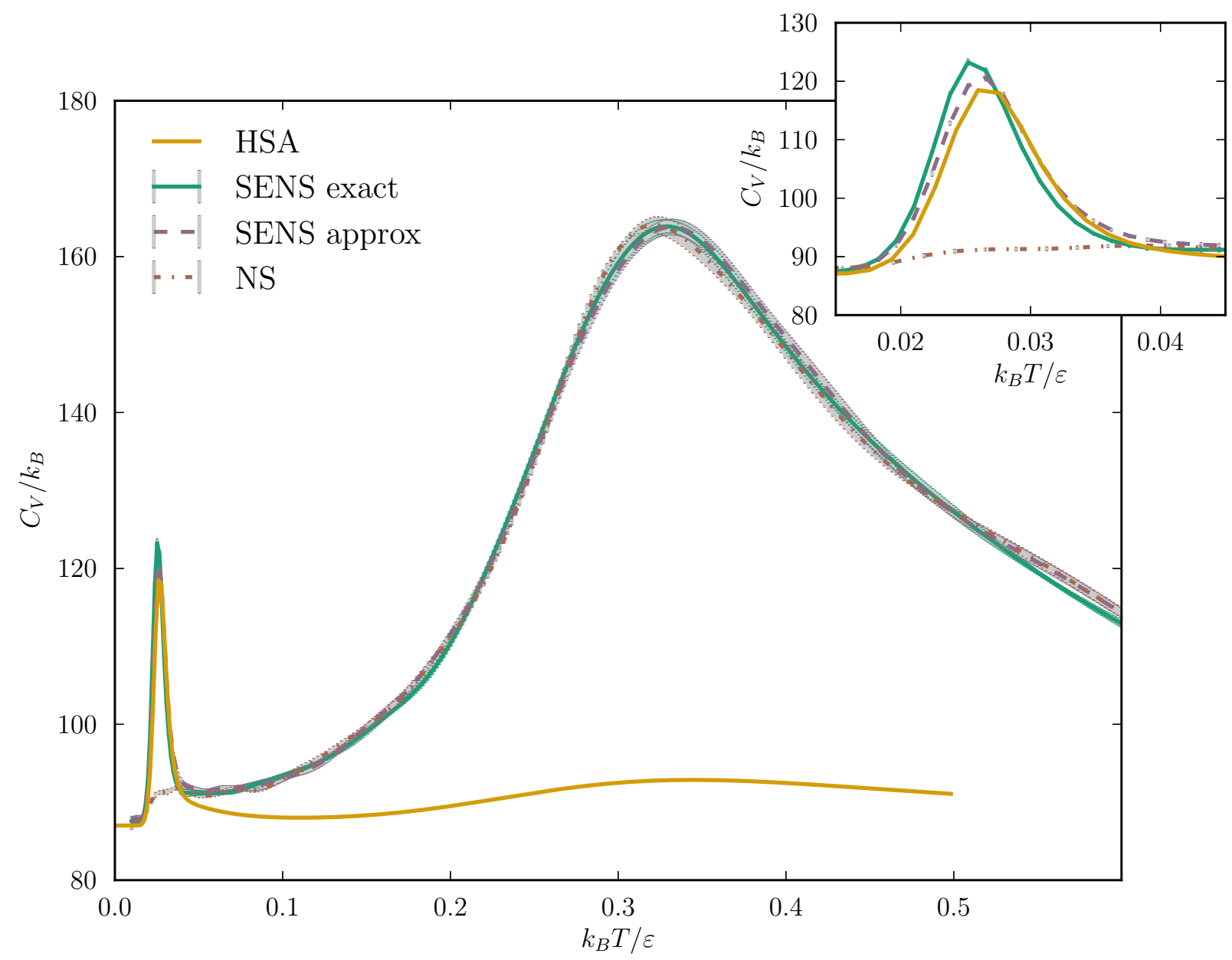

FIG. 6: Heat capacity curves for $\mathrm{LJ}_{31}$. HSA corresponds to the harmonic superposition approximation. All NS and SENS calculations were performed using $K=20000$ replicas.

low temperature peak is completely absent. In Fig. 7 we show a comparison of PT, HSA and exact SENS for a range of replica numbers, see Table I for details. SENS can accurately reproduce the low temperature features of the heat capacity for as low as $K=2500$ replicas, representing an improvement in performance of 20 times over PT [89]. The benefits are not as great for $\mathrm{LJ}_{75}$ where even reaching the region where the HSA becomes accurate enough for swaps to be accepted is challenging.

An approximate version of the method has been proposed to alleviate this problem: similar in spirit to the basin-sampling approach ( $\S$ VII), approximate-SENS interpolates between the high energy density of states (DOS) obtained by NS and the low energy DOS provided by the HSA. Once at low energy, this interpolation can be achieved by starting a fraction of the MC walks from a local minimum configuration sampled from the precomputed collection of minima weighted by 


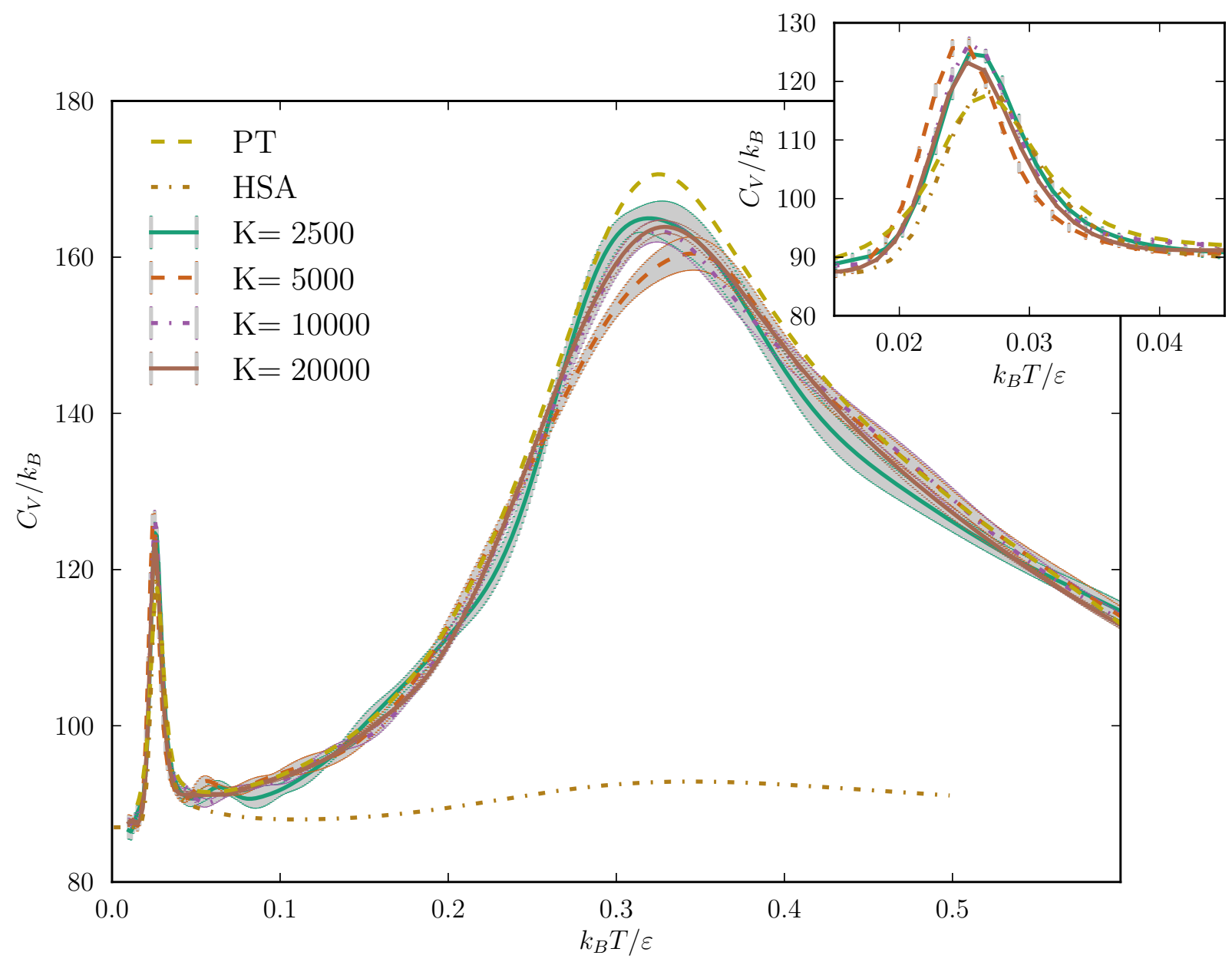

FIG. 7: Comparison of heat capacity curves for $\mathrm{LJ}_{31}$ obtained by exact SENS using different numbers of replicas. The PT and HSA curves were obtained by parallel tempering and the harmonic superposition approximation, respectively. Figure reproduced from [89].

their HSA volume. The transition from the conventional sampling at high energy to the enhanced sampling at low energy is controlled by an onset function. The choice of the energy at which the transition should occur is practically the only additional parameter when compared to exact SENS and NS. Approximate-SENS is easy to implement and generally yields equally good or better results than exact SENS, although it is formally biased.

The thermodynamic analysis of $\mathrm{LJ}_{75}$ by approximate-SENS converges in about $O\left(10^{11}\right)$ energy evaluations, unlike PT, which has been shown to never converge on conventional simulation timescales. The SENS methods are therefore another viable solution to tackle systems exhibiting broken ergodicity. 


\begin{tabular}{lccc}
\hline \multicolumn{4}{c}{$\mathrm{LJ}_{31}$} \\
\hline Method & $K$ & $N$ & $N_{\mathrm{E}}^{(\text {total })}$ \\
\hline \hline PT & & $1.9 \times 10^{11}$ \\
NS ref.[87] & 280000 & $3.4 \times 10^{12}$ \\
SENS approx & 20000 & 10000 & $1 \times 10^{11}$ \\
SENS exact & 20000 & 10000 & $1 \times 10^{11}$ \\
SENS exact & 10000 & 10000 & $5.2 \times 10^{10}$ \\
SENS exact & 5000 & 10000 & $2.6 \times 10^{10}$ \\
SENS exact & 2500 & 10000 & $1.3 \times 10^{10}$ \\
\hline
\end{tabular}

TABLE I: Comparison of methods used to obtain the $\mathrm{LJ}_{31}$ heat capacity curves shown in Figs. 6 and 7. $N_{\mathrm{E}}^{(t o t a l)}$ indicates the total number of energy evaluations (summed over all processors). PT was performed using 24 replicas spread geometrically through the temperature range 0.0125 to 0.6 . Note that, although not shown, approximate SENS can perform as well as exact SENS when fewer replicas are used, results for $\mathrm{LJ}_{75}$ illustrate clearly the capabilities of the method [89]. $N_{E}^{(t o t)}=N \times \mathcal{P} \times N_{\text {iter }}$ is the total number of energy evaluations, where $N$ is the length of each Monte Carlo walk, $\mathcal{P}$ is the number of processors used and $N_{\text {iter }}$ the total number of nested sampling iterations.

\section{BASIN-SAMPLING}

The recently described [44] basin-samping procedure is perhaps the most efficient scheme currently available for sampling global thermodynamic properties. This approach combines knowledge of low-lying local minima on the potential energy landscape with parallel tempering, to connect densities of states that are relevant in the low and high temperature regimes. A twodimensional histogram of probabilities is constructed using the instantaneous potential energy and the energy of local minima obtained by regular quenching. An approximate anharmonic functional form is then fitted to the results corresponding to energy bins for the distribution of local minima on the landscape. This construction has the additional benefit that it can be employed to calculate the potential energy density of both local minimum structures and permutation-inversion isomers, as well as estimates of the total number number of structures and isomers on the landscape.

The regular quenching, which does not affect the instantaneous coordinates in the Markov chain, provides statistics for the number of visits to quench potential energy bin $q$ with potential 


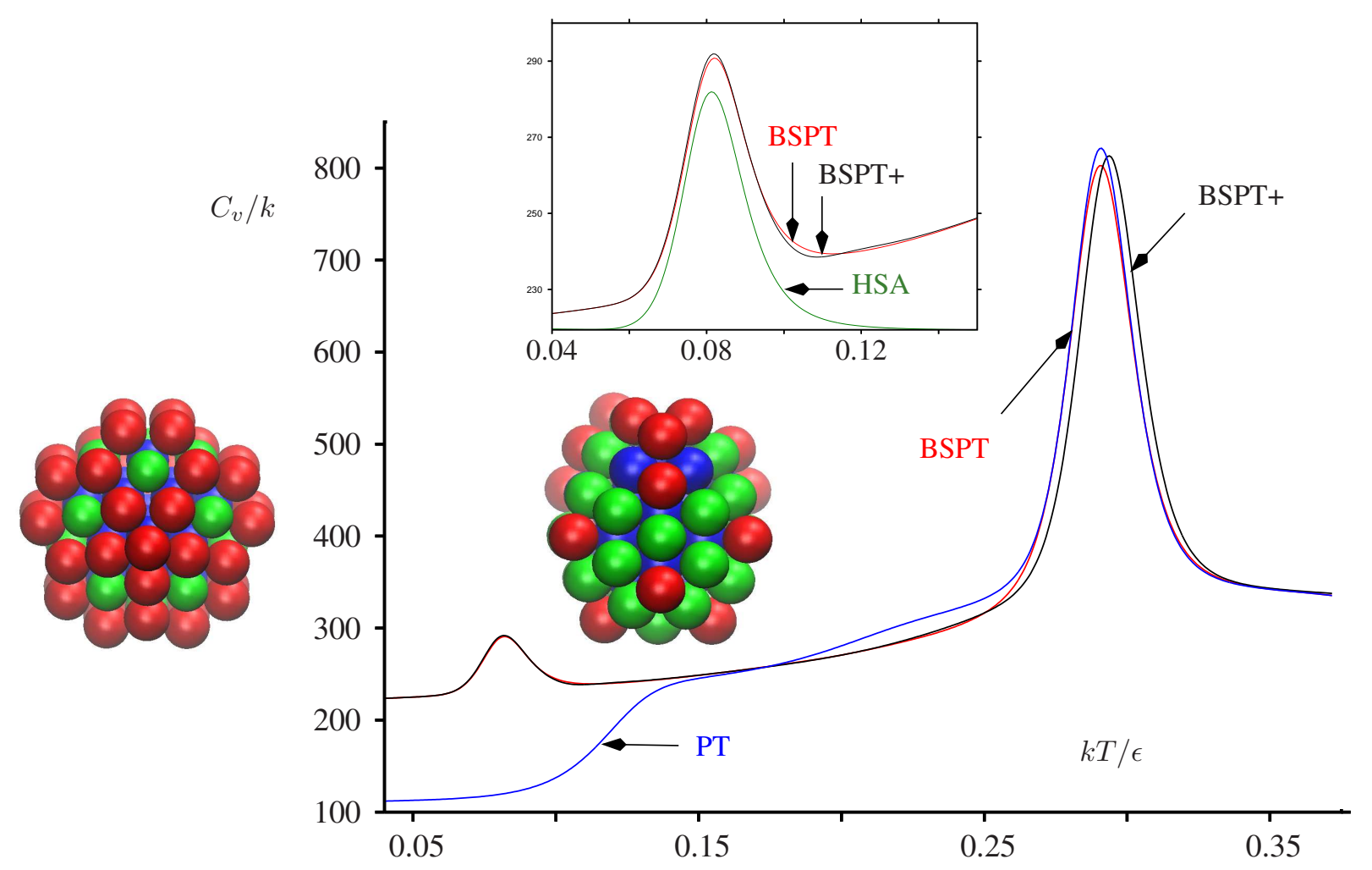

FIG. 8: Heat capacity as a function of reduced temperature for $\mathrm{LJ}_{75}$ [44]. The lowest and second-lowest minima based on a Marks decahedron and an incomplete Mackay icosahedron are illustrated on the left and right of the low-temperature heat capacity peak, respectively. The atoms are coloured according to their contribution to the total energy: the most tightly bound atoms are blue, the least tightly bound are red, with intermediate binding energies in green. The curve marked PT is for the parallel tempering data only from the BSPT run. The inset shows a magnification of the low-temperature peak corresponding to the solid-solid transition and a comparison with the harmonic superposition result. The curves marked BSPT+ result when the original statistics are combined with longer runs aimed at converging the potential energy densities of local minima, and provide a consistency check.

energy $V_{q}^{Q}$ from instantaneous potential energy bin $i$ in replica $r$, denoted by $\mathcal{N}_{i q r}$. The canonical probability distribution

$$
\bar{P}\left(V_{i}^{I}, V_{q}^{Q}, T_{r}\right)=\mathcal{N}_{i q r} / \mathcal{N}_{r} \propto \bar{\Omega}_{c}\left(V_{i}^{I}, V_{q}^{Q}\right) e^{-V_{i}^{I} / k_{B} T_{r}}
$$

is then obtained by minimising

$$
\chi_{2 \mathrm{D}}^{2}=\sum_{r} \sum_{i} \sum_{q} \mathcal{N}_{i q r}\left[\ln \bar{\Omega}_{c}\left(V_{i}^{I}, V_{q}^{Q}\right)-\ln \left(\frac{\mathcal{N}_{i q r} e^{V_{i}^{I} / k_{B} T_{r}} Z_{c}\left(T_{r}\right)}{\mathcal{N}_{r}}\right)\right]^{2},
$$




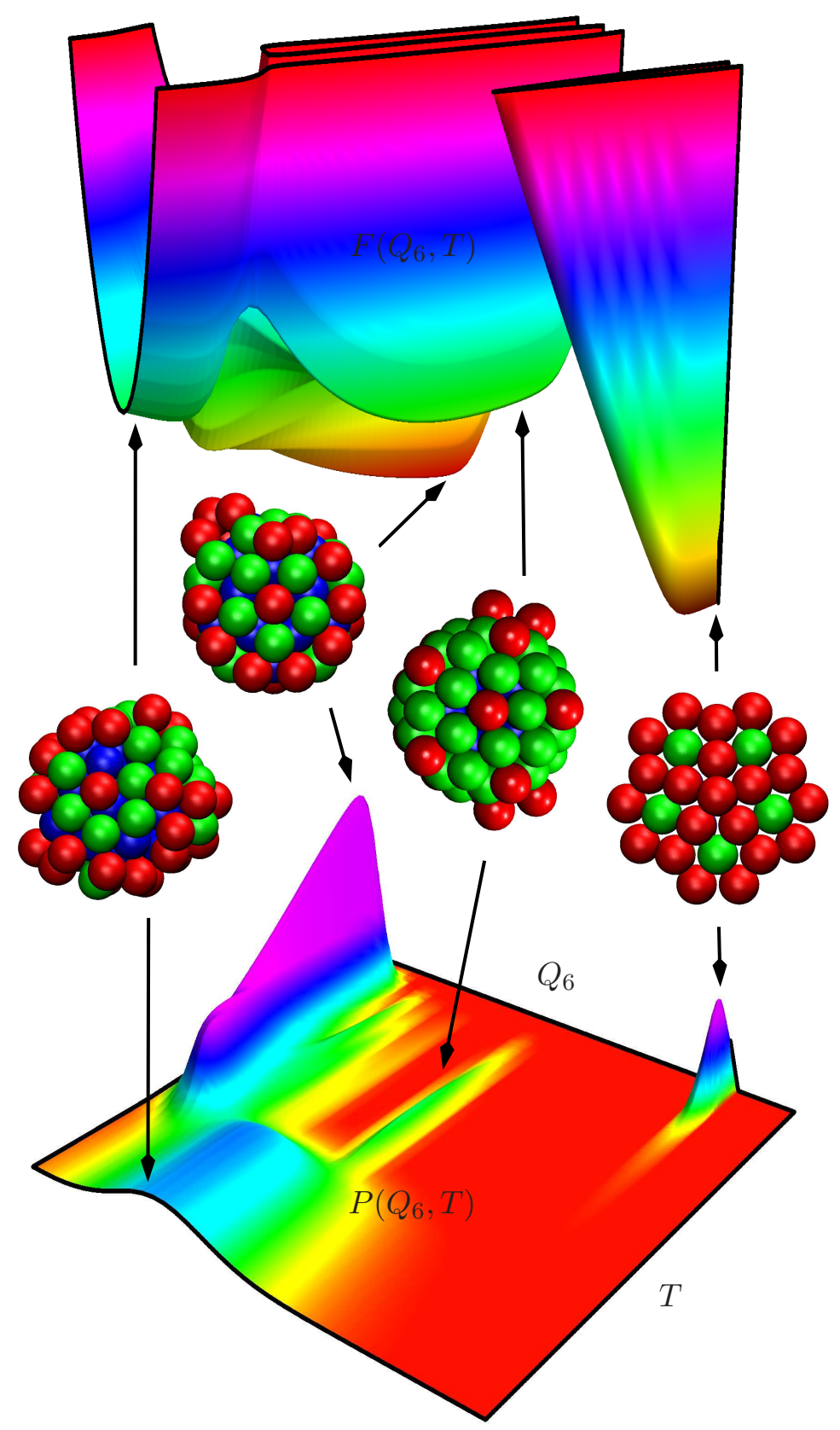

FIG. 9: $\mathrm{LJ}_{75}$ probability distribution $P\left(Q_{6}, T\right)$ and the corresponding free energy surface $F\left(Q_{6}, T\right)=$ $-k_{B} T \ln P\left(Q_{6}, T\right)$ for a database containing 8,391,630 structures [44]. Four structures are illustrated, namely the global minimum (decahedron, $Q_{6}=0.31$ ), the lowest minima based upon icosahedral packing with anti-Mackay and Mackay overlayers [92] $\left(Q_{6}=0.02\right.$ and 0.15 , respectively), and a minimum associated with the liquid-like phase $\left(Q_{6}=0.10\right)$. 


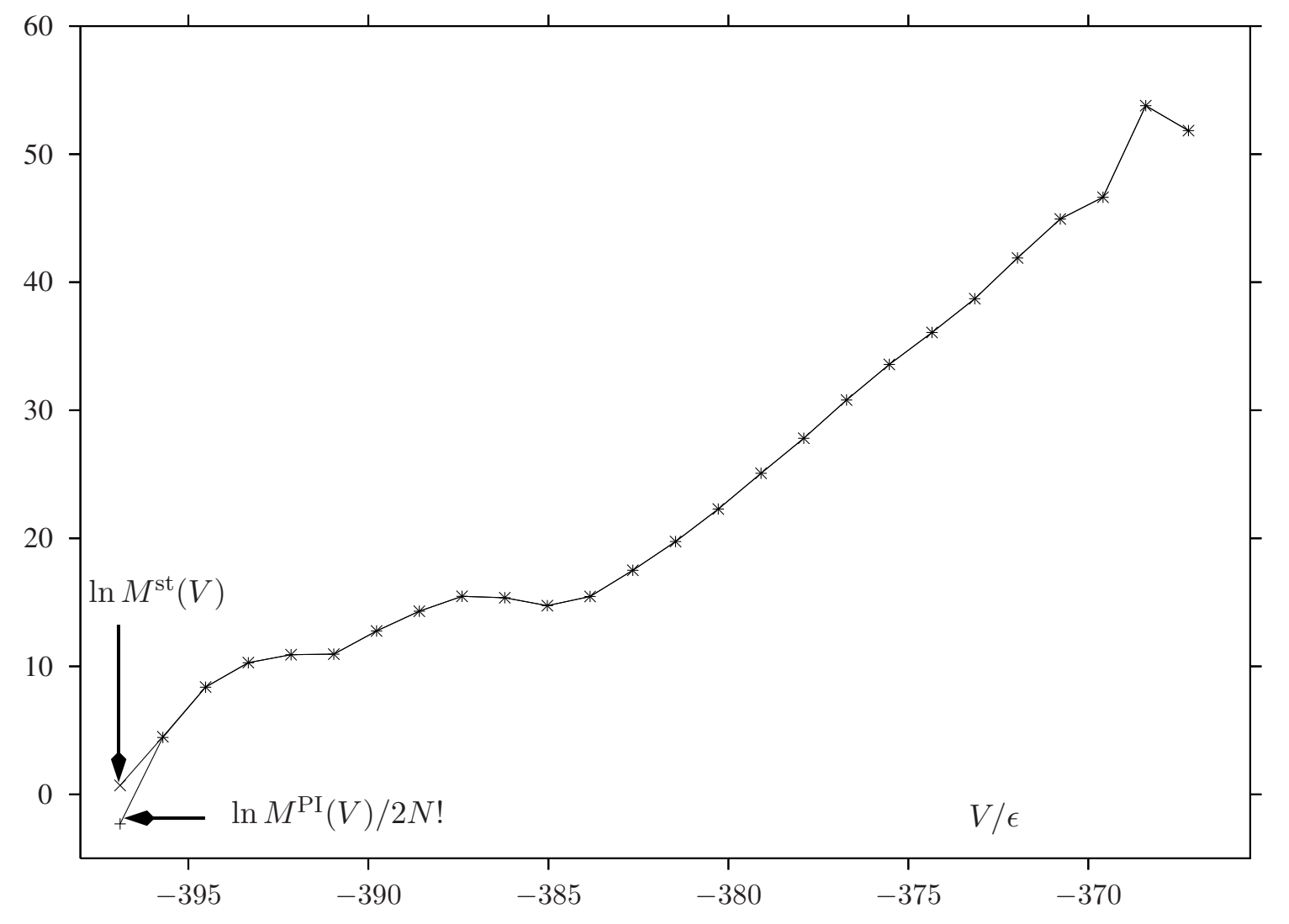

FIG. 10: Calculated values for the $\ln M^{\text {st }}(V)$ and $\ln M^{\mathrm{PI}}(V) / 2 N$ ! as a function of potential energy for $\mathrm{LJ}_{75}[44] . M^{\text {st }}(V)$ and $M^{\mathrm{PI}}(V)$ are the potential energy densities of distinct local minima structures and permutation-inversion isomers, respectively.

where the variables are $\bar{\Omega}_{c}\left(V_{i}^{I}, V_{q}^{Q}\right)$ if we fix $Z_{c}\left(T_{r}\right)=Z_{c}^{*}\left(T_{r}\right)$ from an initial 1D fit to the parallel tempering results for the distribution $P(V, T) \propto \Omega_{c}(V) e^{-V / k_{B} T}$. Here $\Omega_{c}(V)$ is again the configurational density of states. For each replica $r$ with temperature $T_{r}$ we count the number of visits, $\mathcal{N}_{i r}$, to potential energy bins indexed as $V_{i}^{I}$, providing the estimate for $P\left(V_{i}^{I}, T_{r}\right)$ as

$$
P\left(V_{i}^{I}, T_{r}\right)=\mathcal{N}_{i r} / \mathcal{N}_{r}
$$

where $\mathcal{N}_{r}=\sum_{i} \mathcal{N}_{i r}$ is the total number of Monte Carlo steps for replica $r$. We then minimise

$$
\chi_{1 \mathrm{D}}^{2}=\sum_{r} \sum_{i} \mathcal{N}_{i r}\left[\ln \Omega_{c}\left(V_{i}^{I}\right)-\ln \left(\frac{\mathcal{N}_{i r} e^{V_{i}^{I} / k_{B} T_{r}} Z_{c}\left(T_{r}\right)}{\mathcal{N}_{r}}\right)\right]^{2},
$$

where the variables are $\Omega_{c}\left(V_{i}^{I}\right)$ and $Z_{c}\left(T_{r}\right)$. The optimal values are denoted by $\Omega_{c}^{*}\left(V_{i}^{I}\right)$ and $Z_{c}^{*}\left(T_{r}\right)$. In the present work, all the fitting was conducted by minimising $\chi_{2 \mathrm{D}}^{2}$ or $\chi_{1 \mathrm{D}}^{2}$ using the modified limited memory Broyden-Fletcher-Goldfarb-Shanno (L-BFGS) algorithm [93, 94] from the GMIN code [95]. 
To simplify the fitting a model anharmonic density of states was employed for each quench bin, and an efficient representation was obtained using two fitting parameters, $A_{q}$ and $B_{q}$, with

$$
\ln \bar{\Omega}_{c}\left(V_{i}^{I}, V_{q}^{Q}\right)=\left(\kappa^{\prime}+e^{A_{q}} V_{i q}\right) \ln V_{i q}+B_{q},
$$

where $\kappa^{\prime}=\kappa / 2-1$ and $V_{i q}=V_{i}^{I}-V_{q}^{Q}$, the difference between the instantaneous and quench potential energies. Here we have built on previous work [40] that used the analytic density of states for a Morse potential [96]. For $V_{i q} \rightarrow 0$ we recover the usual harmonic result: $\bar{\Omega}_{c}\left(V_{i}^{I}, V_{q}^{Q}\right) \propto$ $V_{i q}^{\kappa / 2-1} . A_{q}$ makes no contribution in this limit, and can therefore be identified with the effective well anharmonicity, while $B_{q}$ incorporates the landscape entropy in terms of the number of minima included in quench bin $q$. This interpretation facilitates the calculation of distributions for the potential energy density of minimum energy structures [44].

Optimal values for the two parameters in each bin were again obtained by fitting, and are denoted $A_{q}^{*}$ and $B_{q}^{*}$. Then to exploit additional information corresponding to the low temperature limit a normal mode analysis of the local minima obtained from basin-hopping global optimisation was used. $B_{q}^{*}$ values were simply replaced up to a specified potential energy threshold using the normal mode data, and the final configurational density of states, $\Omega_{c}\left(V_{i}^{I}\right)$, was obtained by combining the results of the one- and two-dimensional fitting procedures as:

$$
\Omega_{c}\left(V_{i}^{I}\right)=\sum_{q}\left[\delta_{q} \Omega_{c}^{*}\left(V_{i}^{I}\right) \frac{\mathcal{N}_{i q}}{\mathcal{N}_{i}}+\left(1-\delta_{q}\right) \bar{\Omega}_{c}^{*}\left(V_{i}^{I}, V_{q}^{Q}\right)\right],
$$

where the mixing parameter $\delta_{q}=\left(i-i_{\min }^{q}\right) /\left(i_{q}^{\max }-i_{q}^{\min }\right)$ depends on the optimal fitting range for the $q$ bins [44].

Extensive tests were conducted for both $\mathrm{LJ}_{31}$ and $\mathrm{LJ}_{75}$. Some results for the larger cluster are illustrated in Figures 8, 9, and 10. Here $Q_{6}$ is a bond-order parameter [97, 98], which takes larger values of the decahedral global minimum than for minima based upon icosahedral packing. 32 temperature replicas were used, exponentially spaced in the temperature range 0.15 to 0.375 . The production run of $200 \times 10^{6}$ standard parallel tempering steps followed by $60 \times 10^{6}$ BSPT steps (PT with quenches every 30 steps) required 22 hours of wall clock time. When the lowest 13 minima are used to replace the fitted $B_{q}^{*}$ values the resulting heat capacity curve appears to be well converged (Figure 8, with the characteristic low temperature solid-solid peak well reproduced. This result can be compared with previous simulations using an auxiliary harmonic superposition reference [7], which required $3 \times 10^{9}$ steps. In contrast, even $10^{11}$ Monte Carlo steps are not enough to converge the heat capacity using adaptive exchange parallel tempering $[8,91]$. The 
corresponding probability distribution $P\left(Q_{6}, T\right)$ and free energy surface are shown in Figure 9, where various features corresponding to different families of structures can be associated with peaks that are separated in these two-dimensional projections. The calculated potential energy distributions of distinct structures and permutation-inversion isomers are shown in Figure 10. Here the effect of point group symmetry in reducing the number of permutation-inversion isomers is visible at low energy. There is also an unexpected feature, namely a shallow minimum in the distributions around $V=-385 \epsilon$, which has been associated with regions of the potential energy landscape that are relatively sparsely populated [44]. The total number of distinct local minimum structures for this cluster is estimated at around $4 \times 10^{25}$ [44]. The capability of basin-sampling to yield estimates like this as a by-product of the sampling could be particularly interesting for amorphous systems in future studies.

\section{CONCLUSIONS}

The idea of exploiting knowledge of low-lying local minima to enhance sampling $[1,5]$ recently reached fruition in a variety of new techniques [7, 38, 44, 68, 89]. The foundation for each of these methods is the superposition approach, in which the total partition function is decomposed into contributions from distinct local minima [8, 14, 34-37]. The continuing development of these methods provides important cross-validations of results for challenging systems that exhibit broken ergodicity. These results are not only important in themselves, but also provide an essential platform for optimising efficiency, which is likely to come from hybrid schemes, and may be system dependent. Nevertheless, many general conclusions should hold for very diverse problems in chemical physics, and important tests in molecular simulation and soft and condensed matter problems should reveal that further progress is possible.

The authors gratefully acknowledge financial support from the EPSRC and the ERC. S.M acknowledges financial support from the Gates Cambridge Scholarship. We also thank Gábor Csányi for helpful discussions.

[1] R. Zhou and B. J. Berne, J. Chem. Phys. 107, 9185 (1997).

[2] U. H. E. Hansmann and Y. Okamoto, Curr. Op. Struct. Biol. 9, 177 (1999).

[3] F. Wang and D. P. Landau, Phys. Rev. Lett. 86, 2050 (2001). 
[4] W. Watanabe and W. P. Reinhardt, Phys. Rev. Lett. 65, 3301 (1990).

[5] I. Andricioaei, J. E. Straub and A. F. Voter, J. Chem. Phys. 114, 6994 (2001).

[6] J. Kim, J. E. Straub and T. Keyes, Phys. Rev. Lett. 97, 050601 (2006).

[7] V. A. Sharapov and V. A. Mandelshtam, J. Phys. Chem. A 111, 10284 (2007).

[8] V. A. Sharapov, D. Meluzzi and V. A. Mandelshtam, Phys. Rev. Lett. 98, 105701 (2007).

[9] J. Kim, T. Keyes and J. E. Straub, J. Chem. Phys. 135, 061103 (2011).

[10] J. Kim, J. E. Straub and T. Keyes, J. Phys. Chem. B 116, 8646 (2012).

[11] P. J. Ortoleva, T. Keyes and M. Tuckerman, J. Phys. Chem. B 116, 8335 (2012).

[12] G. A. Huber and S. Kim, Biophys. J. 70, 97 (1996).

[13] D. Bhatt, B. W. Zhang and D. M. Zuckerman, J. Chem. Phys. 133, 014110 (2010).

[14] D. J. Wales, Energy Landscapes, Cambridge University Press, Cambridge (2003).

[15] D. J. Wales, Curr. Op. Struct. Biol. 20, 3 (2010).

[16] D. J. Wales, Phil. Trans. Roy. Soc. A 370, 2877 (2012).

[17] D. Frenkel and B. Smit, Understanding molecular simulation, second edition, Academic Press, London (2002).

[18] J. E. Jones and A. E. Ingham, Proc. R. Soc. A 107, 636 (1925).

[19] D. J. Wales and J. P. K. Doye, J. Phys. Chem. A 101, 5111 (1997).

[20] J. P. K. Doye, D. J. Wales and M. A. Miller, J. Chem. Phys. 109, 8143 (1998).

[21] J. P. K. Doye, M. A. Miller and D. J. Wales, J. Chem. Phys. 110, 6896 (1999).

[22] F. Calvo, J. P. Neirotti, D. L. Freeman and J. D. Doll, J. Chem. Phys. 112, 10350 (2000).

[23] J. P. Neirotti, F. Calvo, D. L. Freeman and J. D. Doll, J. Chem. Phys. 112, 10340 (2000).

[24] P. A. Frantsuzov and V. A. Mandelshtam, Phys. Rev. E 72, 037102 (2005).

[25] C. Predescu, P. A. Frantsuzov and V. A. Mandelshtam, J. Chem. Phys. 122, 154305 (2005).

[26] H. Liu and K. D. Jordan, J. Phys. Chem. A 107, 5703 (2003).

[27] L. T. Wille, in Annual Reviews of Computational Physics VII, edited by D. Stauffer, World Scientific, Singapore (2000).

[28] D. J. Wales, Mol. Phys. 100, 3285 (2002).

[29] C. Dellago, P. G. Bolhuis and D. Chandler, J. Chem. Phys. 108, 9236 (1998).

[30] D. Passerone and M. Parrinello, Phys. Rev. Lett. 87, 108302 (2001).

[31] W. E, W. Ren and E. Vanden-Eijnden, Phys. Rev. B 66, 052301 (2002).

[32] P. G. Mezey, Theo. Chim. Acta 58, 309 (1981). 
[33] P. G. Mezey, Potential Energy Hypersurfaces, Elsevier, Amsterdam (1987).

[34] F. H. Stillinger and T. A. Weber, Science 225, 983 (1984).

[35] D. J. Wales, Mol. Phys. 78, 151 (1993).

[36] F. H. Stillinger, Science 267, 1935 (1995).

[37] B. Strodel and D. J. Wales, Chem. Phys. Lett. 466, 105 (2008).

[38] T. V. Bogdan, D. J. Wales and F. Calvo, J. Chem. Phys. 124, 044102 (2006).

[39] F. Sciortino, W. Kob and P. Tartaglia, J. Phys.: Condens. Matt. 12, 6525 (2000).

[40] J. P. K. Doye and D. J. Wales, J. Chem. Phys. 102, 9659 (1995).

[41] J. P. K. Doye and D. J. Wales, J. Chem. Phys. 102, 9673 (1995).

[42] F. Calvo, J. P. K. Doye and D. J. Wales, J. Chem. Phys. 115, 9627 (2001).

[43] I. Georgescu and V. A. Mandelshtam, J. Chem. Phys. 137, 144106 (2012).

[44] D. J. Wales, Chem. Phys. Lett. 584, 1 (2013).

[45] F. G. Amar and R. S. Berry, J. Chem. Phys. 85, 5943 (1986).

[46] M. K. Gilson and K. K. Irikura, J. Phys. Chem. B 114, 16304 (2010).

[47] F. Calvo, J. P. K. Doye and D. J. Wales, Nanoscale 4, 1085 (2012).

[48] Z. Li and H. A. Scheraga, Proc. Natl. Acad. Sci. USA 84, 6611 (1987).

[49] D. J. Wales and H. A. Scheraga, Science 285, 1368 (1999).

[50] K. Klenin, B. Strodel, D. J. Wales and W. Wenzel, Biochimica et Biophysica Acta 1814, 977 (2011).

[51] B. Strodel, J. W. L. Lee, C. S. Whittleston and D. J. Wales, J. Am. Chem. Soc. 132, 13300 (2010).

[52] K. H. Hoffmann, A. Franz and P. Salamon, Phys. Rev. E 66, 046706 (2002).

[53] R. H. Leary and J. P. K. Doye, Phys. Rev. E 60, R6320 (1999).

[54] C. Shang and D. J. Wales, J. Chem. Phys. 141, (2014).

[55] C. Tsallis, J. Stat. Phys. 52, 479 (1988).

[56] C. Tsallis, Braz. J. Phys. 29, 1 (1999).

[57] J. P. K. Doye and D. J. Wales, Phys. Rev. Lett. 80, 1357 (1998).

[58] J. P. K. Doye, D. J. Wales and M. A. Miller, J. Chem. Phys. 109, 8143 (1998).

[59] R. H. Swendsen and J.-S. Wang, Phys. Rev. Lett. 57, 2607 (1986).

[60] D. Earl and M. W. Deem, Phys. Chem. Chem. Phys. 7, 3910 (2005).

[61] Y. Sugita and Y. Okamoto, Chem. Phys. Lett. 329, 261 (2000).

[62] Y. Sugita and Y. Okamoto, Chem. Phys. Lett. 314, 141 (1999).

[63] D. A. Kofke, J. Chem. Phys. 117, 6911 (2002). 
[64] D. A. Kofke, J. Chem. Phys. 120, 10852 (2004).

[65] W. Nadler and U. H. E. Hansmann, Phys. Rev. E 75, 026109 (2007).

[66] W. Nadler and U. H. E. Hansmann, J. Phys. Chem. B 112, 10386 (2008).

[67] R. Denschlag, M. Lingenheil and P. Tavan, Chem. Phys. Lett. 473, 193 (2009).

[68] A. J. Ballard and D. J. Wales, submitted, (2014).

[69] S. Somani, B. J. Killian and M. K. Gilson, J. Chem. Phys. 130, 34102 (2009).

[70] J. G. Kirkwood and E. M. Boggs, J. Chem. Phys. 10, 402 (1942).

[71] J. Hansen and I. McDonald, Theory of Simple Liquids, Third Edition, Academic Press, 3rd edn. (1976).

[72] W. McGill, IEEE Trans. Information Theory 4, 93 (1954).

[73] R. M. Fano, Transmission of Information: A Statistical Theory of Communication, The MIT Press (1961).

[74] B. J. Killian, M. K. Gilson and J. Y. Kravitz, J. Chem. Phys. 127, 24107 (2007).

[75] S. Somani, Conformational sampling and calculation of molecular free energy using superposition approximations, Ph.D. thesis, University of Maryland, College Park (2011).

[76] S. Somani and M. K. Gilson, J. Chem. Phys. 134, 34107 (2011).

[77] C.-E. Chang, M. J. Potter and M. K. Gilson, J. Phys. Chem. B 107, 1048 (Jan. 2003).

[78] Drug Dev. Res. 72, 85 (Feb. 2011).

[79] S. Somani, Y. Okamoto, A. J. Ballard and D. J. Wales, submitted, (2014).

[80] H. M. Berman, J. Westbrook, Z. Feng, G. Gilliland, T. N. Bhat, H. Weissig, I. N. Shindyalov and P. E. Bourne, The protein data bank, The Protein Data Bank home page is http://www.rcsb.org/pdb.

[81] F. H. Allen, Acta Cryst. 58, 380 (Jun 2002).

[82] J. Skilling, Bayesian Analysis 1, 833 (2006).

[83] S. J. Vigeland and M. Vallisneri, Mon. Not. R. Astron. Soc. 440, 1446 (2014).

[84] D. M. Kipping, D. Nesvorny, L. A. Buchhave, J. Hartman, G. A. Bakos and A. R. Schmitt, Astrophysical J. 784, 28 (2014).

[85] N. Pullen and R. J. Morris, Plos One 9, e88419 (2014).

[86] L. B. Pártay, A. P. Bartók and G. Csányi, Phys. Rev. E 89, 022302 (2014).

[87] L. B. Pártay, A. P. Bartók and G. Csányi, J. Phys. Chem. B 114, 10502 (2010).

[88] B. J. Brewer, L. B. Pártay and G. Csányi, Statistics Computing 21, 649 (2011).

[89] S. Martiniani, J. D. Stevenson, D. J. Wales and D. Frenkel, Phys. Rev. X 4, 031034 (2014).

[90] H. Fukunishi, O. Watanabe and S. Takada, J. Chem. Phys. 116, 9058 (2002). 
[91] V. A. Mandelshtam, P. A. Frantsuzov and F. Calvo, J. Phys. Chem. A 110, 5326 (2006).

[92] J. P. K. Doye, M. A. Miller and D. J. Wales, J. Chem. Phys. 111, 8417 (1999).

[93] J. Nocedal, Mathematics of Computation 35, 773 (1980).

[94] D. C. Liu and J. Nocedal, Math. Prog. 45, 503 (1989).

[95] D. J. Wales, Gmin: A program for basin-hopping global optimisation, basin-sampling, and parallel tempering ().

[96] P. C. Haarhoff, Mol. Phys. 7, 101 (1963).

[97] P. J. Steinhardt, D. R. Nelson and M. Ronchetti, Phys. Rev. B 28, 784 (1983).

[98] J. S. van Duijneveldt and D. Frenkel, J. Chem. Phys. 96, 4655 (1992). 